\title{
Erratum
}

\section{A Proof of the Axial Anomaly}

John Lott

Department of Mathematics, Harvard University, Cambridge, MA 02138, USA

Commun. Math. Phys. 97, 371-379 (1985)

Due to a sign error, the paragraph preceding Lemma 3 is in error. In the case of zero ordinary index, one can show independently that the net winding number of the spectrum is equal to $c_{1}$ of the index bundle. The rest of the paper is not affected. I thank S. and V. Della-Pietra for pointing this out.

Communicated by A. Jaffe

Received March 18, 1985 